Preprint of: Ocaña, C. and del Valle, M. "Signal amplification for thrombin impedimetric aptasensor : sandwich protocol and use of gold-streptavidin nanoparticles" in Biosensors and bioelectronics (Ed. Elsevier), vol. 54 (2014), p. 408-414. The final version is available at DOI 10.1016/j.bios.2013.10.068

\title{
Signal amplification for thrombin impedimetric aptasensor: sandwich protocol and use of gold-streptavidin nanoparticles
}

\author{
Cristina Ocaña and Manel del Valle* \\ Sensors and Biosensors Group, Department of Chemistry, Universitat Autonoma de Barcelona, \\ 08193 Bellaterra, Spain \\ *Email:manel.delvalle@uab.cat
}

\begin{abstract}
In this work, we report a highly specific amplification strategy demonstrated for the ultrasensitive biosensing of thrombin with the use of gold-streptavidin nanoparticles (StrepAuNPs) and silver reduction enhancement. The biotinylated aptamer of thrombin was immobilized onto avidin-graphite epoxy composite (AvGEC) electrode surface by affinity interaction between biotin and streptavidin; electrochemical impedance measurements were performed in a solution containing the redox marker ferrocyanide/ferricyanide. The change in interfacial charge transfer resistance (Rct) experimented by the redox marker, was recorded to confirm aptamer complex formation with target protein, thrombin (Thr), in a labeless first stage. A biotinylated second thrombin aptamer, with complementary recognition properties was then used in a sandwich approach. The addition of strep-AuNPs and silver enhancement treatment led to a further increment of Rct thus obtaining significant signal amplification. The Apt1-Thr-Apt2 sandwich formation was inspected by confocal microcopy after incubation with streptavidin quantum dots. In order to visualize the presence of gold nanoparticles, the same silver enhancement treatment was applied to electrodes already modified with the nanoparticlessandwich conjugate, allowing direct observation by scanning electron microscopy (SEM). Results showed high sensitivity and selectivity for thrombin detection, with an improvement from ca.4.7 $\mathrm{pM}$ in a simple assay, to $0.3 \mathrm{pM}$ in the amplified reported scheme.
\end{abstract}

Keywords : impedance, nanoparticles, aptamer, biosensor, thrombin, quantum dots. 


\section{Introduction}

Aptamers are synthetic nucleic acids (DNA or RNA) which selectively bind to lowmolecular-weight organic or inorganic substrates or to macromolecules such as proteins (Jayasena 1999). The affinity constant of aptamers toward their substrates lies in the micromolar to nanomolar range, comparable to the binding constants of antibodies to antigens (Jenison et al. 1994). The interest in aptamers as specific binding agents originates from the relative ease of their preparation by an evolutionary selection procedure that eliminates the need for sophisticated design of the receptor units. Not surprisingly, aptamer have found growing interest as active separation materials in chromatography (Kotia et al. 2000), and electrophoresis (Clark and Remcho 2002), as therapeutics (Biesecker et al. 1999; Hicke et al. 2001) and diagnostic agents, and as active materials for biosensing (Evtugyn et al. 2012). The uses of aptamers for biosensing is particulary interesting, as aptamers could substitute antibodies in bioanalytical sensing and reveal obvious advantages over immunosensors. Aptamer of thrombin is the most known and used aptamer. Thrombin is the last enzyme protease involved in the coagulation cascade, and converts fibrinogen to insoluble fibrin which forms the fibrin gel, both in physiological conditions and in a pathological thrombus (Holland et al. 2000). Therefore, thrombin plays a central role in a number of cardiovascular diseases (Burgering et al. 1997), and it is thought to regulate many processes such as inflammation and tissue repair at the blood vessel wall. Concentration levels of thrombin in blood are very low, and levels down to picomolar range are associated with disease; because of this, it is important to be able to assess this protein concentration at trace level (Centi et al. 2007).

In recent years, there has been great interest in the development of aptasensors. Aptasensors are biosensors that use aptamers as the biorecognition element. This type of biosensor can be classificated, depending on the technique employed for transduction, 
into optical (Lee and Walt 2000), piezoelectric (Srijanto et al. 2012) and electrochemical types (Ocaña et al. 2012; Radi et al. 2005). Electrochemical aptasensors make use of electrochemical transduction for biosensing. Recently, among the different electrochemical techniques available, electrochemical impedance spectroscopy (EIS) (McDonald 1987), has been used in numerous studies of protein detection (Lisdat and Schafer 2008; Radi et al. 2005). This technique is very sensitive to change in the interfacial properties of the modified electrodes caused by biorecognition events at the electrode surface (Bardea et al. 1999; Loo et al. 2012). For this reason, EIS is becoming an attractive electrochemical tool for numerous applications such as immunosensing (Loo et al. 2012), genosensing (Bonanni et al. 2010), enzyme activity determinations (Li et al. 2012), studies of corrosion (Blin et al. 2007) or other surface phenomena (Liao et al. 2007).

In this work, we reported a highly specific amplification strategy demonstrated for the ultrasensitive detection of thrombin through the use of gold-streptavidin nanoparticles and silver enhancement treatment. The transducer employed consisted of an avidin graphite-epoxy composite electrode (Av-GEC), of general use in our laboratories and already extensively studied and applied to amperometric, enzymatic, immuno and genosensing assays (Bonanni et al. 2006; Merkoci et al. 2005; Pumera et al. 2005). The uneven surface of the avidin graphite-epoxy electrode allows the immobilization of biotinylated aptamer of thrombin (AptThrBio1) on its surface by affinity interaction between biotin and avidin. The change of interfacial charge transfer resistance $\left(R_{c t}\right)$ experimented by the redox marker, was recorded to confirm aptamer sandwich formation with target protein, thrombin (Thr).Then, a biotinylated second thrombin aptamer (AptThrBio2), with complementary recognition properties was next used in a sandwich approach. The addition of strep-AuNPs and silver enhancement treatment led to a further increment of $\mathrm{R}_{\mathrm{ct}}$ thus obtaining significant signal amplification, high sensitivity and selectivity. 


\section{Experimental}

\subsection{Chemicals}

Potassium dihydrogen phosphate, potassium ferricyanide $\mathrm{K}_{3}\left[\mathrm{Fe}(\mathrm{CN})_{6}\right]$, potassium ferrocyanide $\mathrm{K}_{4}\left[\mathrm{Fe}(\mathrm{CN})_{6}\right]$, , sodium monophosphate, streptavidin-gold nanoparticles, $655 \mathrm{~nm}$ streptavidin quantum dots (stre-QDs) and the target protein thrombin (Thr), were purchased from Sigma (St. Louis, MO, USA). Poly(ethylene glycol) (PEG), sodium chloride and potassium chloride were purchased from Fluka (Buchs, Switzerland). LI silver enhancement kit was obtained from Nanoprobes (Yaphank, New York).All-solid-state electrodes (AvGECs) were prepared using $50 \mu \mathrm{m}$ particle size graphite powder (Merck, Darmstadt, Germany),Epotek H77 resin and its corresponding hardener (both from Epoxy Technology, Billerica, MA, USA), and avidin. All reagents were analytical reagent grade. Aptamers used in this study were purchased by TIBMOLBIOL (Berlin, Germany). Stock solutions of were diluted with sterilized and deionised water, separated into fractions and stored at $-20{ }^{\circ} \mathrm{C}$ until required. Their bases sequences were:

- AptThrbio1: 5'-GGTTGGTGTGGTTGG-Biotin-3'

- AptThrbio2: 5'-Biotin-AGTCCGTGGTAGGGCAGGTTGGGGTGACT-3'

- AptCytc:5'-Biotin-

AGTGTGAAATATCTAAACTAAATGTGGAGGGTGGGACGGGAAGAAG TTTATTTTTCACACT-3'

All solutions were made up using MilliQ water from MilliQ System (Millipore, Billerica, MA, USA). The buffers employed were: PBS (187 mM NaCl, $2.7 \mathrm{mM} \mathrm{KCl}$, 
8.1 $\mathrm{mM} \mathrm{Na}_{2} \mathrm{HPO}_{4} \cdot 2 \mathrm{H}_{2} \mathrm{O}, 1.76 \mathrm{mM} \mathrm{KH}_{2} \mathrm{PO}_{4}, \mathrm{pH}$ 7.0) and $10 \mathrm{nM}$ PBS without $\mathrm{NaCl}$ and $\mathrm{KCl}$.

\subsection{Apparatus}

AC impedance measurements were performed with Autolab PGStat 20 (Metrohm Autolab B.V, Utrecht, The Netherlands). Fra (Metrohm Autolab) software was used for data acquisition and control of the experiments. A three electrode configuration was used to perform the impedance measurements: a platinum-ring auxiliary electrode (Crison 52-67 1, Barcelona, Spain), an $\mathrm{Ag} / \mathrm{AgCl}$ reference electrode and the constructed Av-GEC as the working electrode. Temperature-controlled incubations were done using an Eppendorf Thermomixer 5436. A scanning electron microscope (SEM) (Merlin, Zeiss, Germany) and a confocal microscope (SP5, Leica, Solms, Germany) were used to visualize silver enhanced strep-AuNPs and strep-QDs respectively on electrode surface.

\subsection{Construction of working electrodes}

The electrodes were prepared using a PVC tube body (6 $\mathrm{mm}$ i.d.) and a small copper disk soldered at the end of an electrical connector, as shown on Figure 1(a). The working surface was an avidin epoxy-graphite conductive composite, formed by a mixture of graphite (18\%), avidin (2\%) and epoxy resin $(80 \%)$, deposited on the cavity of the plastic body(Bonanni et al. 2007). The composite material was cured at $40{ }^{\circ} \mathrm{C}$ for 7 days. Before each use, the electrode surface was moistened with MilliQ water and then thoroughly smoothed with abrasive sandpaper and finally with alumina paper (polishing strips 301044-001, Orion) in order to obtain a reproducible electrochemical surface (Lermo et al. 2007; Williams et al. 2003).

\subsection{Procedure}


The scheme of experimental procedure, described in detail below, is represented in Figure 1.

$<$ Figure 1 $>$

\subsubsection{Aptamer immobilization}

The first step consists of aptamer immobilization onto the electrode surface.160 $\mu \mathrm{L}$ of aptamer solution in MilliQ water at the desired concentration was heated to $80-90{ }^{\circ} \mathrm{C}$ for $3 \mathrm{~min}$ to promote the loose conformation of the aptamer. Then, the solution was dipped in a bath of cold water and the electrode was immersed in it, where the avidinbiotin affinity interaction took place for $15 \mathrm{~min}$. This was followed by two washing steps using PBS buffer solution for $10 \mathrm{~min}$, in order to remove unadsorbed aptamer.

\subsubsection{Blocking step}

After that, to minimize any possible nonspecific adsorption, the electrodes were dipped in $160 \mu \mathrm{L}$ of PEG $40 \mathrm{mM}$ for $15 \mathrm{~min}$ (Ocaña et al. 2012). This was followed by two washing steps using PBS buffer solution for $10 \mathrm{~min}$

\subsubsection{Thrombin detection}

The electrodes were dipped in a solution with the desired concentration of Thr. The incubation took place for $15 \mathrm{~min}$. Then, the biosensors were washed twice with PBS buffer solution for $10 \mathrm{~min}$.

\subsubsection{Sandwich formation}

In order, to realized the aptamer sandwich formation, the electrodes were dipped in a solution containing AptThrBio2. The incubation took place for 15 minutes. This was followed by two washing steps using PBS buffer solution for 10 minutes. 


\subsubsection{Addition of strep-QDs}

Av-GEC electrodes modified with the aptamer sandwich were incubated in a solution of $40 \mathrm{nM}$ of strep-QDs for 20 minutes at $25^{\circ} \mathrm{C}$ with stirring. Then the electrodes were washed twice with $10 \mathrm{mM}$ PBS buffer. Negative controls were performed for the strepQDs addition step either using AptCytc as no complementary target

\subsubsection{Addition of strep-AuNPs}

Av-GEC electrodes modified with sandwich complex were incubated in an eppendorf tube containing a solution of strep-gold nanoparticles at the desired concentration in PBS buffer. The tube was incubated at $25^{\circ} \mathrm{C}$ with gentle stirring for 20 minutes. This step was followed by two gentle washing steps in PBS buffer for 10 minutes at $25^{\circ} \mathrm{C}$. Negative controls were performed for the strep-AuNPs addition step either using AptCytc as non complementary target.

2.4.7 Silver enhancement of strep-AuNPs

$20 \mu \mathrm{l}$ of a solution obtained by the combination of equal volumes of enhancer and iniciator were deposited onto the electrode surface and left for 7 minutes to facilitate the reaction. The electrodes were then thoroughly washed with deionized water to stop the reaction. The silver enhancing solution was always prepared immediately before use. For silver enhancement treatment, the negative control consisted of using nonbiotinylated complementary target.

All incubations were carried out at controlled temperature in the thermomixer.

\subsection{Impedimetric detection}

Impedance experiments were carried out at an applied potential of $0.17 \mathrm{~V}$ (vs. $\mathrm{Ag} / \mathrm{AgCl}$ reference electrode), with a range of frequency of $50 \mathrm{KHz}-0.05 \mathrm{~Hz}$, an $\mathrm{AC}$ amplitude of $10 \mathrm{mV}$ and a sampling rate of 10 points per decade above $66 \mathrm{~Hz}$ and 5 points per decade 
at the lower range. All measurements were performed in PBS buffer containing $0.01 \mathrm{M}$ $\mathrm{K}_{3}\left[\mathrm{Fe}(\mathrm{CN})_{6}\right] / \mathrm{K}_{4}\left[\mathrm{Fe}(\mathrm{CN})_{6}\right]$ (1:1) mixture, used as a redox marker. The impedance spectra were plotted in the form of complex plane diagrams (Nyquist plots, $-Z_{\text {im }} v s . Z_{\text {re }}$ ) and fitted to a theoretical curve corresponding to the equivalent circuit with $Z_{\text {view }}$ software (Scribner Associates Inc., USA). The equivalent circuit was formed by one resistor/ capacitor element in series with a resistance. The parameter $\mathrm{R}_{1}$ corresponds to the resistance of the solution, $R_{2}$ is the charge transfer resistance (also called $R_{c t}$ ) between the solution and the electrode surface, whilst CPE is associated with the double-layer capacitance (due to the interface between the electrode surface and the solution). The use of a constant phase element (CPE) instead of a capacitor is required to optimize the fit to the experimental data, and this is due to the nonideal nature of the electrode surface. For all performed fitting, the chi-square goodness-of-fit test was thoroughly checked to verify calculations. In all cases, calculated values for each circuit remained in the range of $0.0003-0.15$ much lower than the tabulated value for 50 degrees of freedom (67.505 at 95\% confidence level). In this work, we focused on the variation of change resistance transfer $\left(R_{c t}\right)$. In order to compare the results obtained from the different electrodes used, and to obtain independent and reproducible results, relative signals are needed (Bonanni et al. 2006). Thus, the $\Delta_{\text {ratio }}$ value was defined according to the following equations:

$\Delta_{\text {ratio }}=\Delta_{\mathrm{s}} / \Delta_{\mathrm{p}}$

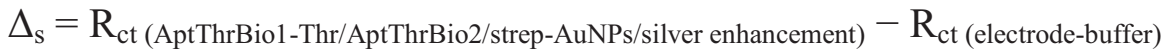

$\Delta_{\mathrm{p}}=\mathrm{R}_{\mathrm{ct} \text { (AptThrBiol) }}-\mathrm{R}_{\mathrm{ct}}$ (electrode-buffer)

where $R_{c t}$ (AptThrBio1-Thr/AptThrBio2/strep-AuNPs/silver enhancement) was the electron transfer resistance value measured after aptamer sandwich formation; $\mathrm{R}_{\mathrm{ct}}$ (AptThrBiol) was the electron transfer resistance value measured after aptamer inmobilitation on the electrode, and $R_{\text {ct (electrode-buffer) }}$ was the electron transfer resistance of the blank electrode and buffer. 


\section{Results}

\subsection{EIS characterization}

$<$ Figure 2>

In Figure 2, the EIS spectrum obtained for a biosensing experiment using aptamer sandwich protocol and two amplification steps are shown. Impedance measurements were performed before modifying the electrode surface (bare Av-GEC electrode), after modifying the electrode surface with aptThrbio1,Thr, aptThrBio2 (sandwich formation), strep-AuNPs and finally after silver deposition. As shown in this figure, Rct increased after any modification of the electrode surface. This can be due to the increased difficulty of the redox reaction of $\left[\mathrm{Fe}(\mathrm{CN})_{6}\right]^{3-4-}$ to take place, due to the sensor surface alteration (Katz and Willner 2003). Two different factors may be take into account to explain that: the electrostatic repulsion and the sterical hindrance. The first one is more significant in the first step of protocol; when the aptThrbiol is immobilizated onto the electrode surface by avidin-biotin affinity, a first layer is formed, where negatively charged phosphate groups of aptamer skeleton are responsible of the electrical repulsion towards the negatively charged redox marker, thus inhibiting the interfacial transfer process and resulting in Ret increment. The addition of Thr and a second apThrBio 2 to form a sandwich results in further increment of Rct due to the improved quantity of negative charge and to the hindrance caused by the formation of a double layer. In this work, strep-AuNPs and silver enhancement treatment were used with the aim of amplifying the EIS sandwich formation signal. After the addition of strep-AuNPs we can observe an increment of Rct value because of the increased space resistance due to Au-streptavidin conjugates. At working $\mathrm{pH} 7$, streptavidin is slightly negatively chraged 
( $\mathrm{pI}$ is around $\mathrm{pH} 5$ ) and this fact also contribute to enhance the resistance (Sivasankar et al. 1998). In the second amplification step, silver enhancement treatment (Cai et al. 2002; Hanaee et al. 2007), also a significant increment of Rct value was observed attributable to silver deposition on gold.

3.2. Analytical perfomance of the aptasensor for detection of thrombin

$<$ Figure 3 $>$

$<$ Table 1 $>$

After the optimization of aptThrBiol and PEG concentration, the proposed aptasensor using sandwich protocol and strep-AuNPs and silver enhancement treatment as the amplification label is challenged with different concentration of Thr. Fig. 3a showed different calibration curves and Fig.3b) their respectives regression curves; aptThrBio1-Thr, (2) Sandwich formation between aptThrbio1, thr and aptThrBio2, (3) aptamer sandwich modified with strep-AuNPs, and (4) aptamer sandiwich modified with strep-AuNPs and silver enhancement treatment. In previous works about DNA hybridization after silver enhancement treatment was not possible to obtain good impedimetric measurements (Bonanni et al. 2008). In this case, although the reproducibility was not excellent, the calibration curve obtained showed a good RSD. As we can be seen in fig. 3 a), all calibration curves increased until the value of $100 \mathrm{pM}$ of Thr, this could be due to that more concentration of $100 \mathrm{pM}$ causes a saturation of the sensor surface. Moreover, it was observed a signal increment of $89 \%$ between aptThrbio1-Thr, simple biosensing, and sandwich formation with strep-AuNPs and silver enhancement treatment. As it can be observed in table 1, the use of silver enhancement treatment led to a higher sensitivity and signal amplification compared to the others calibration curves obtained. This fact confirmed that the silver deposition on gold nanoparticles causes sterial hindrance and electrostic factor, producing a signal 
increase. Despite a better sensitivity, the detection limit obtained in this case resulted lower than the one obtained with only strep-AuNPs. This could be attributable to the increased process steps increased the error. The best detection limit, around $0.2 \mathrm{pM}$, corresponded to sandwich and strep-AuNPS calibration curve. This confirm that the proposed methods showed low detection limit and ultrahigh sensitivity for the detection of thrombin. Typical impedimetric aptasensors have detections limits between 1-10pM (Frense et al. 2013; Kara et al. 2010; Xu et al. 2013), even in the nanomolar range (Radi et al. 2005).

\subsection{Selectivity of the aptasensor}

$<$ Figure 4 $>$

To verify that the signal is dependent on the specific recognition, our protocol for thrombin detection was evaluated by challenging it with possible interfering proteins present in blood serum, comparing it versus the label-free detection alternative, see Fig 4. From this investigation, we found that the presence of the interference proteins such as albumin, cytochrome c, fibrinogen, prothrombin and immunoglobulin $\mathrm{g}$, at serum concentration level exhibits negligible responses compared with $75 \mathrm{pM} \mathrm{Thr}$, concentrations four or five magnitudes lower than $\mathrm{Thr}$ concentration. These observations suggested that the displacement mechanism relies on specific recognition and that the aptasensor sandwich method was highly specific for the detection of Thr. Moreover, it is important to emphasize that prothrombin protein is a precursor of thrombin in the blood clotting process and this aptasensor is capable of distinguishing both proteins with high selectivity.

3.4. Confocal microscopy characterization

$<$ Figure 5 $>$ 
Aptamer sandwich formation were inspected by confocal microscopy after strep-QDs incubation. The images obtained are shown in figure 3. The first images (fig. 3 a) ) corresponds to the electrodes modified with aptamer sandwich formation and strepQDs. The strong fluorescence observed in this case is due to the presence of the complex formation between strep-QDs and aptamer sandwich by avidin- streptavidin affinity. The non fluorescence observed in the second image (fig.3 b) ) corresponds to the negative control, that did not use biotinylated complementary aptamer.

\subsection{Scanning electron microscope characterization}

In order to visualize the presence and distribution of gold nanoparticles, the silver treatment was used. These experiments provided also an image of the homogeneity and accessibility of the anchorage points supplied by the avidin entrapped in the biocomposite. SEM images taken at an acceleration voltage of $3 \mathrm{kV}$ are shown in Figure 6. Part a, ilustrated a positive experiment with aptamer sandwich and strep-AuNPs. As it can be observed in the figure 6 , the distribution of silver enhanced-gold nanoparticles is quite homogeneous. This means also a regular distribution of avidin molecules in the biocomposite and well-organized formation of aptamer sandwich onto the electrode surface. The formed nanoparticles were monocrystalline. Thanks to the disposition of the streptavidin around of gold nanoparticles, the silver crystallizes in specific directions. Comparing this experiment with negative control that did not use a biotinylated complementary aptamer, part $b$, we could observed a surface without nanoparticles.

\section{Conclusions}

In this work, the signal amplification of impedimetric detection of thrombin was proposed using an aptamer sandwich formation with strep-AuNPs and silver enhancement treatment. The use of strep-AuNPs and strep-QDs permitted rapid 
formation of an easily detected conjugate with biotinylated aptamer sandwich through a streptavidin-biotin interaction. Thanks to signal amplification with strep-AuNPs and silver deposition, it was possible to increase the sensitivity of the method. Besides, for a comparable amount of aptThr-Thr, the signal resulted $89 \%$ amplified, compared to results recorded with simple biosensing AptThr-Thr. Moreover, the limit of detection obtained was of $0.3 \mathrm{pM}$. A good linear range, $0.1-100 \mathrm{pM}$, and high selectivity respect different serum proteins at serum concentration level was also attained with this protocol. A silver enhancement treatment was also performed to visualize the gold nanoparticles on the electrode surface with SEM. This treatment allowed a clear visualization of the particles distribution on the sensor surface. For all, we think that this method could be able to be extended for determination of other proteins and provide a promising potential in clinical application.

\section{Acknowledgments}

Financial support for this work has been provided by Spanish Ministry of Science and Innovation, (MICINN,Madrid) through project CTQ2010-17099 and by the Catalonia program ICREA Academia. Cristina Ocaña thanks the support of Ministry of Science and Innovation (MICINN, Madrid, Spain) for the predoctoral grant.

\section{References}

Bardea, A., Patolsky, F., Dagan, A., Willner, I., 1999. Chem. Comm.(1), 21-22.

Biesecker, G., Dihel, L., Enney, K., Bendele, R.A., 1999. Immunopharmacology 42(1-3), 219-230.

Blin, F., Koutsoukos, P., Klepetsianis, P., Forsyth, M., 2007. Electrochim. Acta 52(21), 6212-6220.

Bonanni, A., Esplandiu, M.J., del Valle, M., 2008. Electrochim. Acta 53(11), 4022-4029.

Bonanni, A., Esplandiu, M.J., del Valle, M., 2010. Biosens. Bioelectron. 26(4), 1245-1251.

Bonanni, A., Esplandiu, M.J., Pividori, M.I., Alegret, S., del Valle, M., 2006. Anal. Bioanal. Chem. 385(7), 1195-1201.

Bonanni, A., Pividori, M.I., del Valle, M., 2007. Anal. Bioanal. Chem. 389(3), 851-861.

Burgering, M.J.M., Orbons, L.P.M., vanderDoelen, A., Mulders, J., Theunissen, H.J.M., Grootenhuis, P.D.J., Bode, W., Huber, R., Stubbs, M.T., 1997. J. Mol. Biol. 269(3), 395-407.

Cai, H., Wang, Y.Q., He, P.G., Fang, Y.H., 2002. Anal. Chim. Acta 469(2), 165-172.

Centi, S., Tombelli, S., Minunni, M., Mascini, M., 2007. Anal. Chem. 79(4), 1466-1473. 
Clark, S.L., Remcho, V.T., 2002. Electrophoresis 23(9), 1335-1340.

Evtugyn, G.A., Kostyleva, V.B., Porfireva, A.V., Savelieva, M.A., Evtugyn, V.G., Sitdikov, R.R., Stoikov, I.I., Antipin, I.S., Hianik, T., 2012. Talanta 102(0), 156-163.

Frense, D., Kang, S., Schieke, K., Reich, P., Barthel, A., Pliquett, U., Nacke, T., Brian, C., Beckmann, D., 2013. Journal of Physics: Conference Series 434(1).

Hanaee, H., Ghourchian, H., Ziaee, A.A., 2007. Anal. Biochem. 370(2), 195-200.

Hicke, B.J., Marion, C., Chang, Y.F., Gould, T., Lynott, C.K., Parma, D., Schmidt, P.G., Warren, S., 2001. J. Biol. Chem. 276(52), 48644-48654.

Holland, C.A., Henry, A.T., Whinna, H.C., Church, F.C., 2000. FEBS Lett. 484(2), 87-91.

Jayasena, S.D., 1999. Clin. Chem. 45(9), 22.

Jenison, R.D., Gill, S.C., Pardi, A., Polisky, B., 1994. Science 263(5152), 1425-1429.

Kara, P., de la Escosura-Muñiz, A., Maltez-da Costa, M., Guix, M., Ozsoz, M., Merkoçi, A., 2010. Biosens. Bioelectron. 26(4), 1715-1718.

Katz, E., Willner, I., 2003. Electroanal. 15(11), 913-947.

Kotia, R.B., Li, L., McGown, L.B., 2000. Anal. Chem. 72(4), 827-831.

Lee, M., Walt, D.R., 2000. Anal. Biochem. 282(1), 142-146.

Lermo, A., Campoy, S., Barbé, J., Hernández, S., Alegret, S., Pividori, M.I., 2007. Biosens. Bioelectron. 22(9-10), 2010-2017.

Li, Y., Syed, L., Liu, J., Hua, D.H., Li, J., 2012. Anal. Chim. Acta 744(0), 45-53.

Liao, Y.-M., Feng, Z.-D., Chen, Z.-L., 2007. Journal of Dentistry 35(5), 425-430.

Lisdat, F., Schafer, D., 2008. Anal. Bioanal. Chem. 391(5), 1555-1567.

Loo, A.H., Bonanni, A., Ambrosi, A., Poh, H.L., Pumera, M., 2012. Nanoscale 4(3), 921-925.

McDonald, J.R., 1987. Impedance Spectroscopy. John Wiley, New York.

Merkoci, A., Aldavert, M., Marin, S., Alegret, S., 2005. Trac-Trends Anal. Chem. 24(4), 341-349.

Ocaña, C., Pacios, M., del Valle, M., 2012. Sensors 12(3), 3037-3048.

Pumera, M., Aldavert, M., Mills, C., Merkoci, A., Alegret, S., 2005. Electrochim. Acta 50(18), 37023707.

Radi, A., Acero Sánchez, J.L., Baldrich, E., O'Sullivan, C.K., 2005. Anal. Chem. 77(19), 6320-6323.

Sivasankar, S., Subramaniam, S., Leckband, D., 1998. Proceedings of the National Academy of Sciences 95(22), 12961-12966.

Srijanto, B.R., Cheney, C.P., Hedden, D.L., Gehl, A.C., Crilly, P.B., Huestis, M.A., Ferrell, T.L., 2012. Sens. Lett. 10(3-4), 850-855.

Williams, E., Pividori, M.I., Merkoçi, A., Forster, R.J., Alegret, S., 2003. Biosens. Bioelectron. 19(3), 165-175.

Xu, H., Gorgy, K., Gondran, C., Le Goff, A., Spinelli, N., Lopez, C., Defrancq, E., Cosnier, S., 2013. Biosens. Bioelectron. 41(0), 90-95. 
Table 1. Summary of calibration results.

\begin{tabular}{ccccc} 
Calibration curve & Regression Curve & $\begin{array}{c}\text { Detection } \\
\text { Limit }\end{array}$ & $\begin{array}{c}\text { Linear } \\
\text { Range }\end{array}$ & $\begin{array}{c}\% \\
\text { Amplification }\end{array}$ \\
\hline AptThrBio1-Thr & $\Delta_{\text {ratio }}=1.042+0.0157[\mathrm{Thr}]$ & $4.7 \mathrm{pM}$ & $0.75-100 \mathrm{pM}$ & - \\
$\begin{array}{c}\text { Aptamer Sandwich } \\
\text { Aptamer }\end{array}$ & $\Delta_{\text {ratio }}=1.495+0.0194[\mathrm{Thr}]$ & $2 \mathrm{pM}$ & $0.75-100 \mathrm{pM}$ & 35 \\
$\begin{array}{c}\text { Aundwich/Strep- } \\
\text { AuNPs }\end{array}$ & $\Delta_{\text {ratio }}=1.874+0.0201[\mathrm{Thr}]$ & $0.2 \mathrm{pM}$ & $0.1-100 \mathrm{pM}$ & 57 \\
$\begin{array}{c}\text { Aptamer } \\
\text { sandwich/strep- } \\
\text { AuNPs/silv.enh }\end{array}$ & $\Delta_{\text {ratio }}=2.477+0.0219[\mathrm{Thr}]$ & & & \\
\hline
\end{tabular}




\section{Figure captions}

Figure 1. Scheme of the preparation of avidin graphite-epoxy composite electrodes and the biosensing procedure.

Figure 2. Nyquist diagrams for EIS measurements of: $\bullet$ bare AvGEC electrode, $\circ$ biotinylated aptamer of thrombin $1, \boldsymbol{\nabla}$ biotinylated aptamer of thrombin and thrombin protein, $\Delta$ sandwich complex, - sandwich complex modified with strep-AuNPs, $\square$ sandwich complex modified with strep-AuNPs and silver enhancement treatment.

Figure 3. a) Calibration curves and b) Regression curves of: - biotinylated aptamer of thrombin 1, ○ sandwich complex, $\square$ sandwich complex modified with strep-AuNPs, sandwich complex modified with strep-AuNPs and silver enhancement treatment.

Figure 4. 3D bar chart of response towards different proteins present in serum, without the amplification protocol and with the sandwich/amplification protocol.

Figure 5. a) Confocal images of: a.1) positive control using biotinylated aptamer of thrombin 1 + thrombin + biotinylated aptamer of thrombin $2+$ strep-QDs, a.2) negative control using biotinylated aptamer of thrombin $1+$ thrombin + biotinylated aptamer of cytocrome $\mathrm{c}+$ strepQDs. b) SEM images of: b.1) experiment using biotinylated aptamer of thrombin $1+$ thrombin + biotinylated aptamer of thrombin $2+$ strep-AuNPs + silver enhancement treatment, b.2) experiment using biotinylated aptamer of thrombin $1+$ thrombin + biotinylated aptamer of cytochrome $\mathrm{c}+$ strep-AuNPs + silver enhancement treatment. SEM images were taken at a acceleration voltage of $3 \mathrm{KV}$ and a resolution of $2 \mu \mathrm{m}$ and Confocal images were collected at $625 \mathrm{~nm}$ and with excitation wavelenght of $405 \mathrm{~nm}$. 


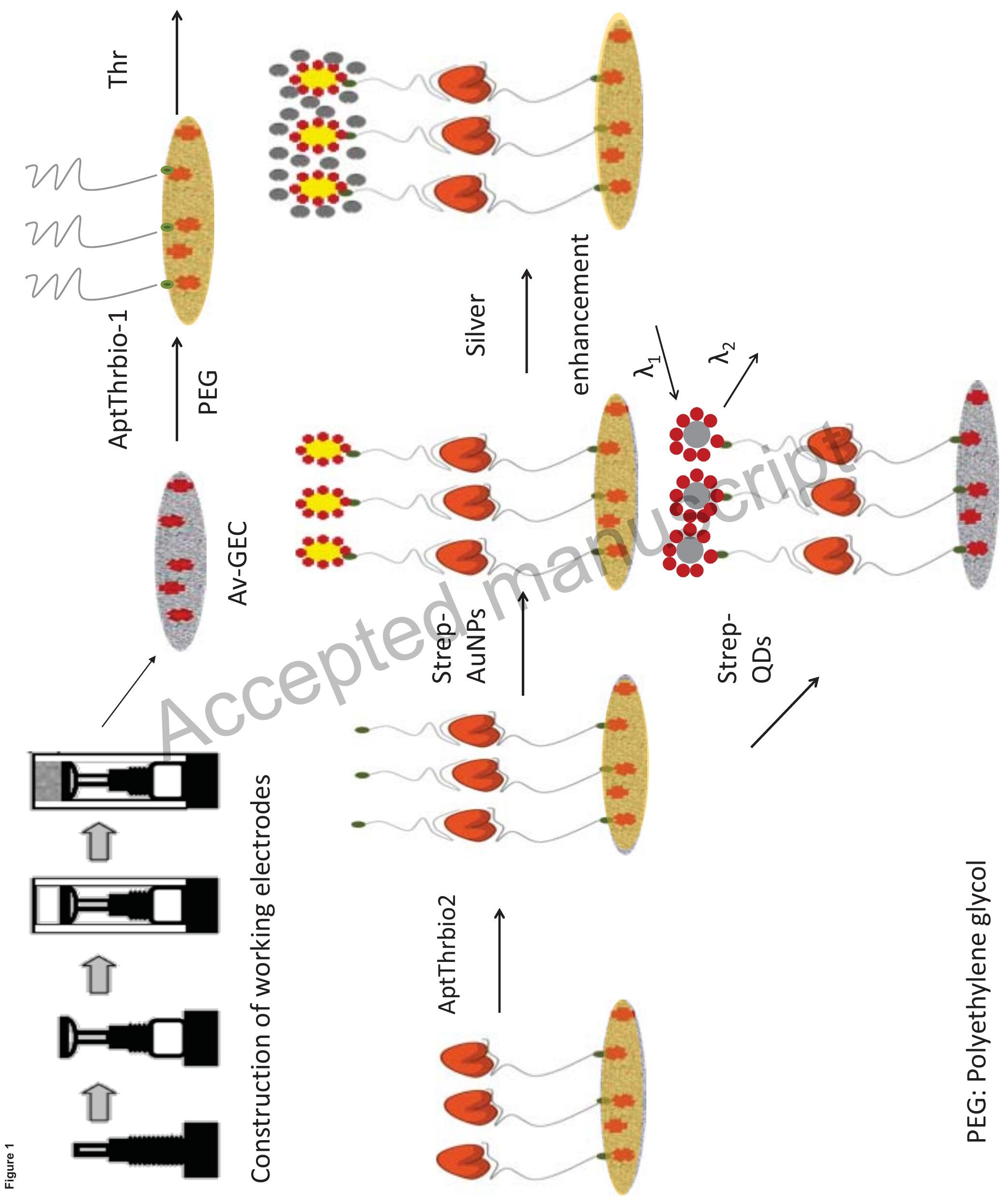




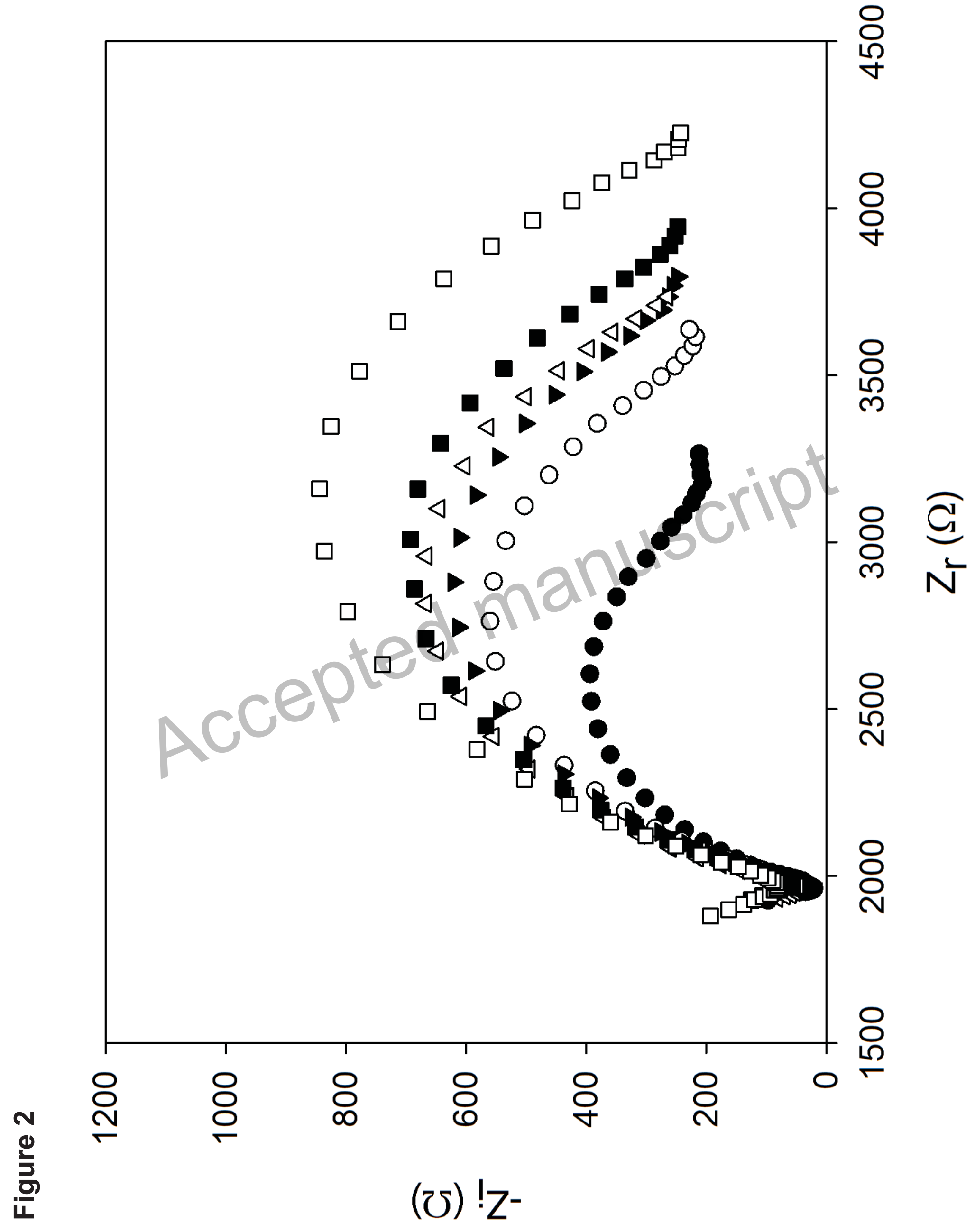


Figure 3

Figure 3.
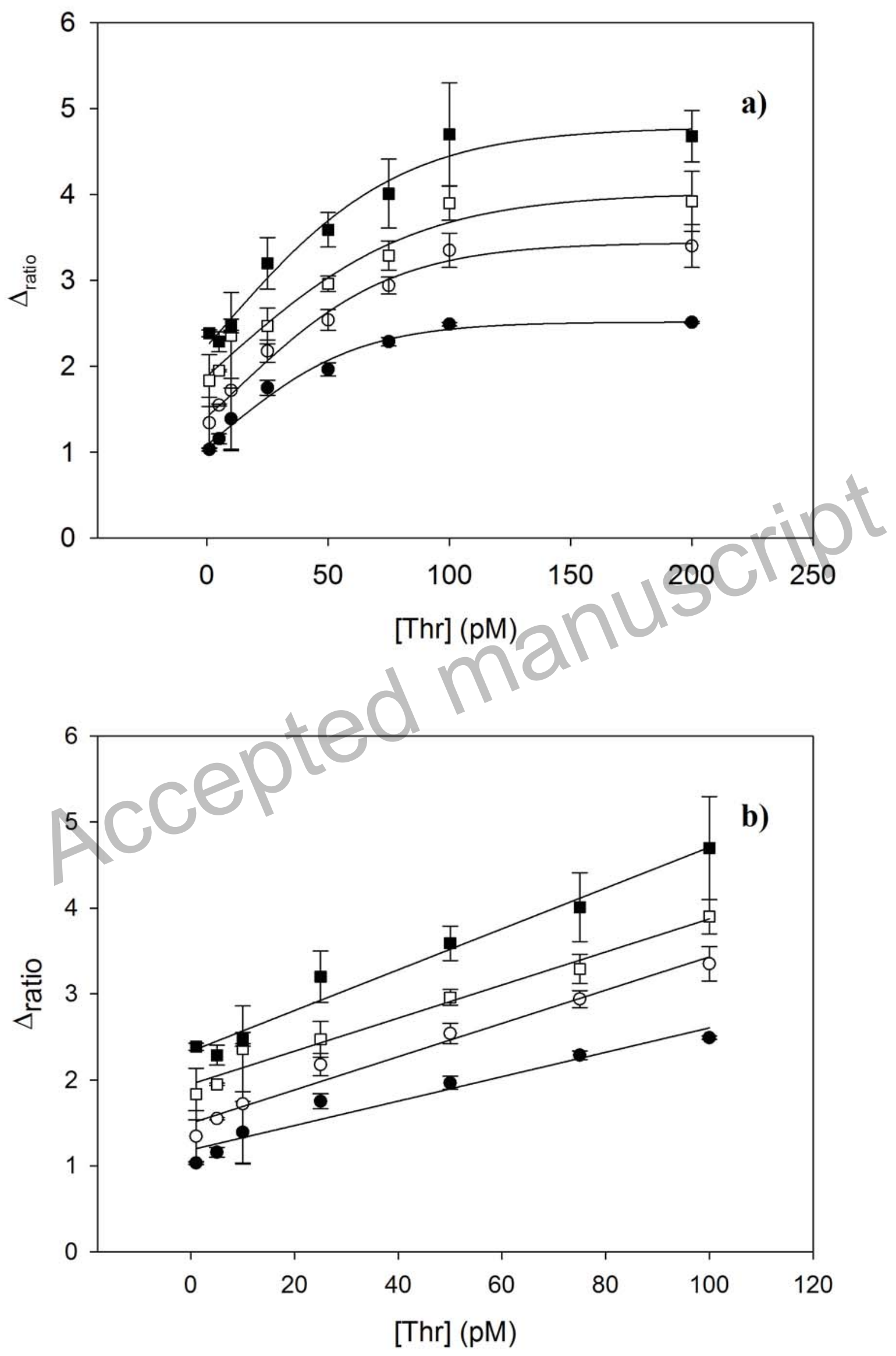


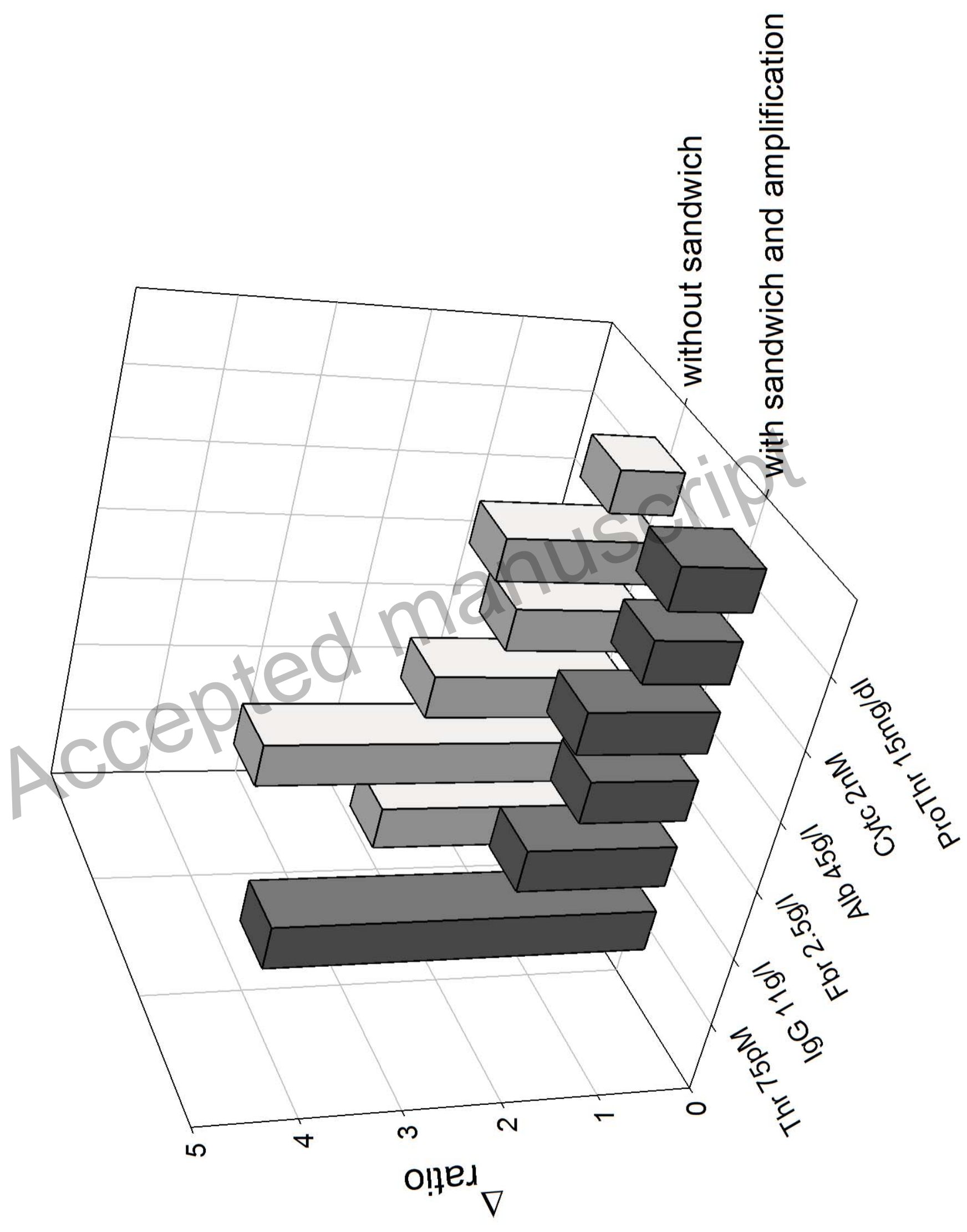



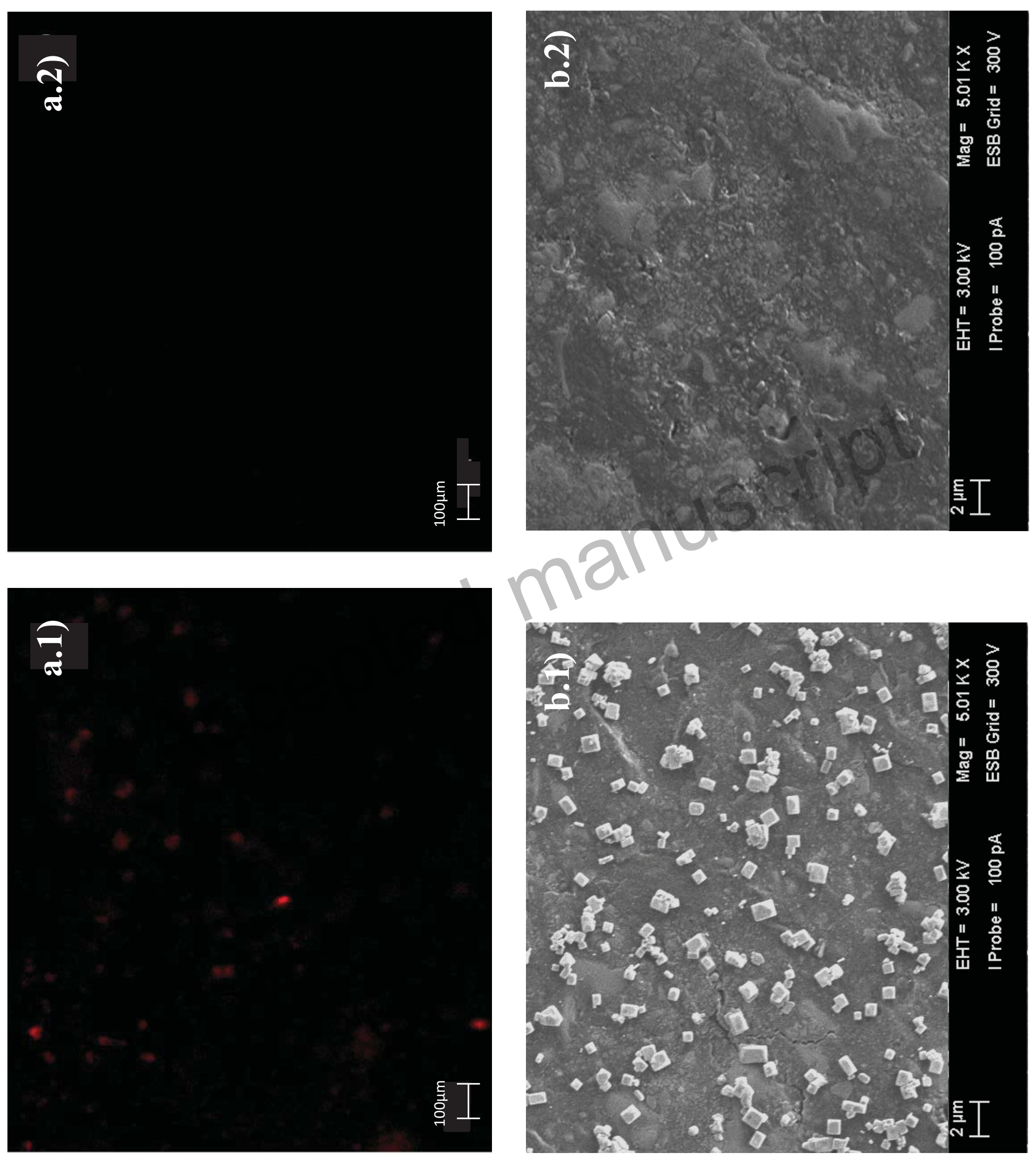

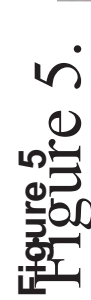

